\title{
The SPHERE exoplanet imager: a new management paradigm?
}

\author{
Francois Wildi $^{* a}$, Jean-Luc Beuzit $^{\mathrm{b}}$, Markus Feldt ${ }^{\mathrm{c}}$, David Mouillet ${ }^{\mathrm{b}}$, Kjetil Dohlen $^{\mathrm{d}}$, Pascal Puget ${ }^{\mathrm{b}}$ \\ ${ }^{a}$ Observatoire de Genève, 51 ch des Maillettes, CH-1290 Sauverny, Switzerland \\ ${ }^{\mathrm{b}}$ Laboratoire d'Astrophysique de Grenoble, B.P. 53, F-38041 Grenoble Cedex 9, France \\ ${ }^{c}$ Max Planck Institut für Astronomie, Königstuhl 17, D-69117 Heidelberg, Germany \\ ${ }^{\mathrm{d}}$ Laboratoire d'Astrophysique de Marseille, B.P. 8, F-13376 Marseille Cedex 12, France
}

\begin{abstract}
The SPHERE is an exo-solar planet imager, which goal is to detect giant exo-solar planets in the vicinity of bright stars and to characterize them through spectroscopic and polarimetric observations. It is built by a consortium of 11 institutes from five countries. Rather than a traditional instrument, it is a complete system with a core made of an extreme-Adaptive Optics (AO) turbulence correction, pupil tracker and interferential coronagraphs feeding 3 different science instruments: working in Near Infrared Y, J, H and Ks bands $(0.95-2.32 \mu \mathrm{m})$ and in visible $(0.5-0.9 \mu \mathrm{m})$ light. In this paper, we focus on project organization matters like the make-up of the consortium and the decision flow. We try to identify if the management is adequate to the size and scope of the project and the consortium that is in charge of developing it. Our view regarding the organization of future multi-site complex fast track projects are presented.
\end{abstract}

Keywords: project management, requirements management, scheduling, extrasolar planets, extreme AO

\section{INTRODUCTION}

\subsection{Project history}

The SPHERE ${ }^{1}$ project is the offspring of the two competing responses to the ESO call for proposals for a VLT planet finder instrument, VLT-PF ${ }^{2}$ and CHEOPS $^{3}$, combining the best of each of them. Before and after kick-off the project has undergone a challenging phase of technical and managerial consolidation. It is a complex system with its extreme $\mathrm{AO}(\mathrm{SAXO})^{4}$, the coronagraphic devices ${ }^{5}$, and the an Infra-Red Dual-beam Imaging and Spectroscopy science module (IRDIS), its Integral Field Spectrograph (IFS $)^{6,7}$ and the $\mathrm{ZIMPOL}^{8}$ dual imaging polarimeter.

\subsection{Science case}

The prime objective of SPHERE is the discovery and study of new planets orbiting stars by direct imaging of the circumstellar environment. The challenge consists in the very large contrast of luminosity between the star and the planet (larger than $\sim 12.5$ magnitudes or $\sim 10^{5}$ flux ratio), at very small angular separations, typically inside the seeing halo. The whole design of SPHERE is therefore optimized towards high contrast performance in a limited field of view and at short distances from the central star. Both evolved and young planetary systems will be detected, respectively through their reflected light (mostly in the Vis by ZIMPOL) and through the intrinsic planet emission in the NIR with IRDIS and the IFS. The two NIR imagers will provide complementary detection capacities and characterization potential, in terms of field of view, contrast, and spectral domain.

SPHERE has both a discovery and a characterization mission. The NIR survey mode is the main observing mode which will be used for $\sim 80 \%$ of the observing time. It combines IRDIS dual band imaging (DBI) in $\mathrm{H}$ band with imaging spectroscopy using the IFS in the Y-J bands. This configuration permits to benefit simultaneously from the optimal capacities of both dual imaging over a large field (out to $\sim 5^{\prime \prime}$ radius) and spectral imaging in the inner region (out to at least $0.9^{\prime \prime}$ radius). In particular, it allows to reduce the number of false alarms and to confirm potential detections obtained in one channel by data from the other channel. This will be a definitive advantage in case of detections very close to the limits of the system.

The NIR characterization mode, in which IRDIS is used alone, will allow observations in Y to short-K, bands in DBI, dual polarimetry, low or medium resolution spectroscopy or in classical imaging. In the visible search and characterization mode, ZIMPOL works alone and offers polarimetric capacities with unique performance in reflected light very close to the star, down to the level required for the first direct detection in the visible of old close-in planets. ZIMPOL also provides classical imaging in the visible, offering unique high-Strehl performance.

* francois.wildi@obs.unige.ch 


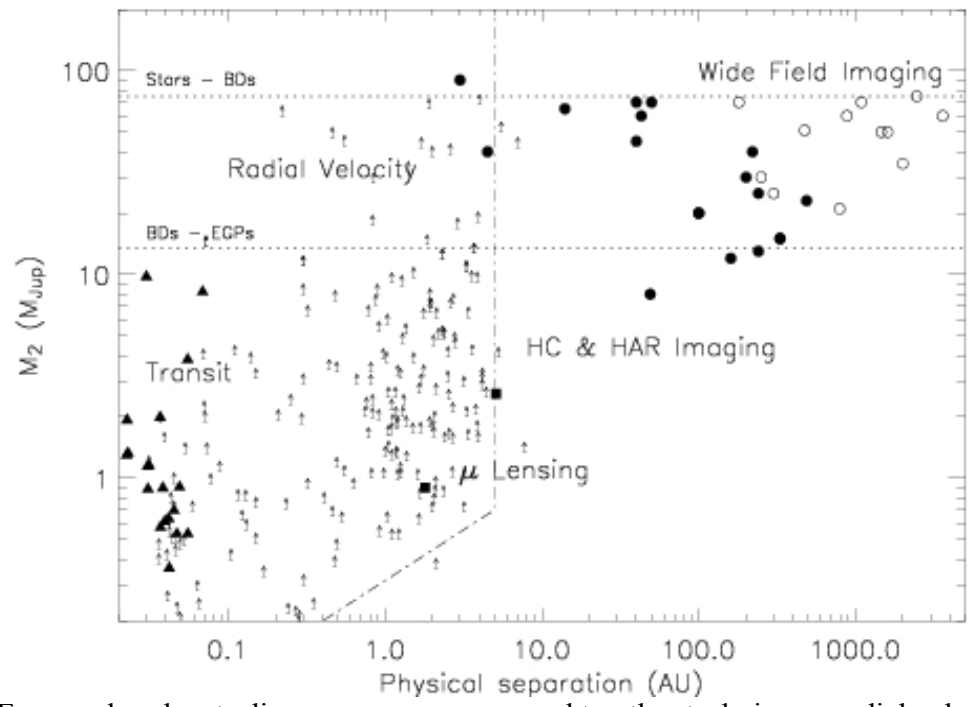

Figure 1. SPHERE exo-solar planets discovery space compared to other techniques: radial velocity (arrows), transit (filled triangles), micro lensing (filled boxes), wide field imaging (open circles) and high contrast and high dynamic range imaging (filled circles)

\section{SYSTEM ARCHITECTURE OVERVIEW}

SPHERE is divided in four subsystems: the Common Path and Infrastructure (CPI) and the three science instruments IRDIS, IFS, and ZIMPOL.

- The CPI optics includes pupil stabilizing fore optics (tip-tilt and rotation), the SAXO extreme adaptive optics system, a NIR coronagraphic device and a separate visible coronagraphic device. The two NIR instruments (IRDIS and IFS) are fed with the coronagraphed NIR beam and ZIMPOL gets the visible coronagraphed beam, after it has shared the visible photons with the AO wavefront sensor.

- IRDIS (Infra-Red Dual Imaging Spectrograph), the main science channel responsible for wide-field imaging in one or two simultaneous spectral bands or two orthogonal polarisations and low and medium resolution long slit spectroscopy.

- $\quad$ The IFS (Integral Field Spectrograph), working from 0.95 to $1.7 \mu \mathrm{m}$ and providing low spectral resolution (R 30) over a limited, 3" x 3", field-of-view.

- ZIMPOL, the visible dual imaging polarimeter, working between 0.65 and $0.95 \mu \mathrm{m}$. Due to its innovative lock-in technique, it can achieve polarimetric precisions better than $10^{-5}$ on a localized signal measured differentially against a smooth background. 


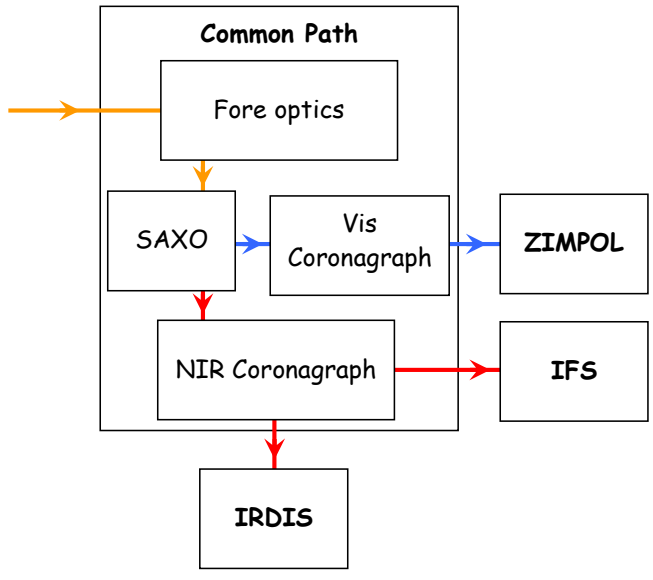

Figure 2. The four sub-systems of SPHERE. Optical beams are indicated in red for NIR, blue for Vis and orange for CPI.

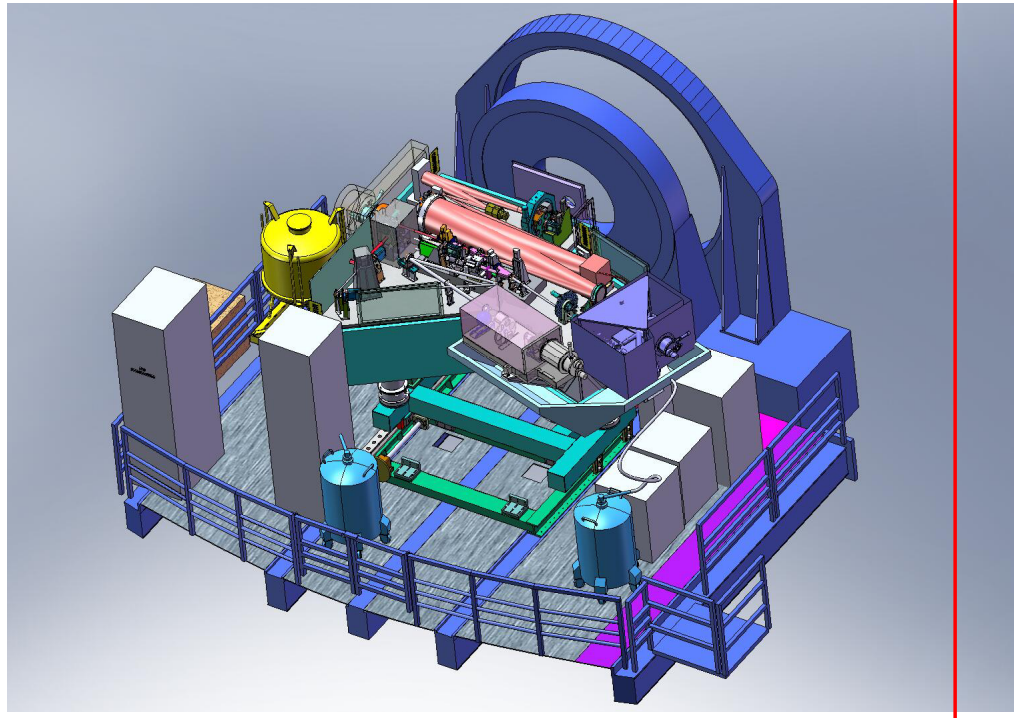

Figure 3. SPHERE on the Nasmyth platform of the VLT. Status at OFDR

The common path will be mounted on a large actively damped optical bench to which each science instrument will dock as a whole. When in operation on one of the VLT Nasmyth platforms, SPHERE will be entirely enclosed in a thermal/dust cover and include a comprehensive automated cryo-vacuum system supplying 4 cryostats and a separate vacuum container. The current design of SPHERE is shown in Figure 3.

Besides classical optical components, the common path embeds numerous new high-technology components like the high order deformable mirror, toroidal mirrors manufactured by spherical polishing of pre-stressed substrates, a dedicated electron multiplying CCD for wavefront sensing, achromatic 4 quadrants coronagraph, classical and apodized Lyot coronagraphs. A good number of these components have one or more of their degrees of freedom motorized, for a total of around 60 motors.

\section{SPHERE PROJECT ORGANISATION}

\subsection{The SPHERE consortium}

SPHERE is built by a consortium of 11 institutes and ESO which is both the final customer and a formal partner in the project. These institutes are located 5 countries. Not counting the industrial contractors, there are 3 hierarchical levels in the consortium with partners providing components, sub-systems and/or the complete instrument.

The consortium is made of the following entities: of LAOG (Grenoble, France), MPIA (Heidelberg, Germany), LAM (Marseille, France), LESIA (Paris, France), LUAN (Nice, France), INAF (Padova, Italy), Observatoire de Genève (Geneva, Switzerland), ETHZ (Zurich, Switzerland), NOVA (Netherlands), ONERA (Chatillon, Paris) and ASTRON (Netherlands) in collaboration with ESO.

\subsection{History of the consortium}

The consortium is the result of the ESO-induced merger of two proposals that were originally competing in a call for an exo-planet imager. The first one made by a consortium of 6 institutes and the second one by a consortium of 5 institutes. In the merging process, all institutes from both projects have stayed on-board the SPHERE project. Moreover, all science focal planes from both proposals have been integrated in the SPHERE projects...

\subsection{The project organisation}

The head of the project in organised in a manner very classical in the field: It has

- a Principal Investigator (PI) has overall project responsible and is the single point of contact with ESO. There is a co-PI to assist him. 
- a Project Manager (PM) has overall responsibility for the project development and coordination, including technical management, procurement, safety, documentation, and cost and schedule control.

- a project scientist (PS) is responsible for ensuring the overall instrument performance and the agreement between the science objectives and the technical developments.

- a system engineer (SE) is responsible for the overall technical performance of the instrument, for the system analysis and for all AIT activities.

To ensure a fair share of the science return, each institute of the consortium has a co-investigator (Co-I), with direct interest in the primary science goals of the project. These Co-I's will assist the PI and Co-PI for all aspects related to the science.

The task of the SE is very demanding in the optics domain because the instrument has 3 scientific focal planes, very tight wavefront error budgets, top-of-the-line coronagraphy, cosmetics, ghosts etc. To support him the other areas, a system team was created where, on top of the PM, PS and SE, specialists of control, instrument software, and assembly integrations and test are sitting.

A project office was defined which is hardly anything like an office, since it involves people from 5 geographic locations!

Each of the four sub-systems has a complete management structure with PM, SE, PS and involves at least two of the participating institutes (up to 8 institutes for CPI). Moreover, one institute is delocalised on 4 sites hundreds of kilometres away from each others.

For administrative purposes each institute has a local project control and administration responsible (LPCA) who reports to the PM for all activities related to the management of the project (schedule, budget, manpower, resources, etc.)

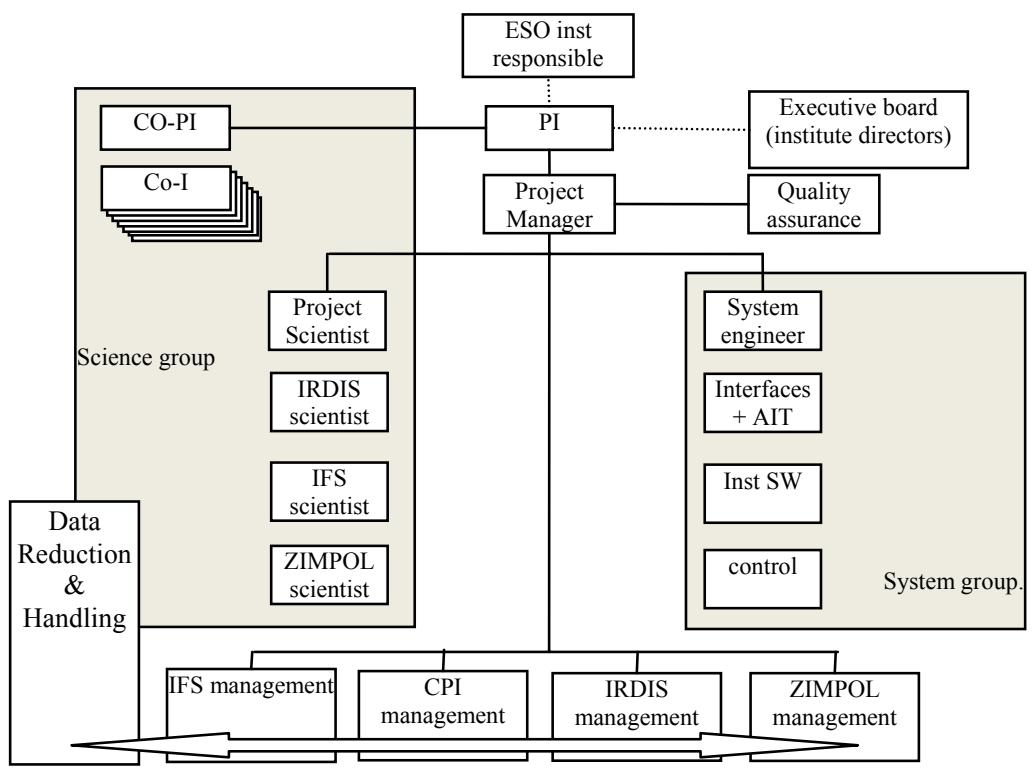

Figure 4. SPHERE project management organization

\subsection{The development logic}

The development of the instrument is driven by a set of Top Level Requirements formed after the two consortia merged. Those requirements are closely associated from the two original science cases that have also undergone a consolidation phase. These requirements are expressed in terms of the mission and they were not deemed to be sufficiently verifiable to be made applicable for the development of the instrument itself. Therefore, a technical specification has been produced where all requirements are explicitly expressed in a way that makes them verifiable. This specification document is the contractual commitment of the consortium towards ESO and it was made in collaboration with ESO which took in charge the specification of all environmental and operational constraints. 


\section{MANAGING THE REQUIRMENTS}

On a typical system, there is a straightforward flow of the requirements from science, to system level specification to sub-system specification. The SPHERE system however is quite complex and the requirements are numerous. In turn these requirements induce an even larger number of sub-system level requirements. Some of those become so stringent that they can lead to very difficult solutions. We observe the need to feed the level of difficulty and/or uncertainty back to the system level so that the requirements can be re-evaluated and possibly traded when needed.

In our project the decision flux is not specified in the management plan. The requirement feedback loop does not formally exist even if de facto, there is always the possibility for anyone to let anybody know that there is a high risk that a requirement might not be reached. We wish we could find a method and/or a tool that would allow to trade the requirements to optimise the design of SPHERE as a whole rather than leaving the freedom of design only to the lower smallest of the units.

\section{SCHEDULING}

\subsection{Practical difficulties}

An essential element of the project management is the establishment and the upkeep of a project schedule. Practically we are using the MS project software. Some tasks are managed directly are the system level but most of them are managed at the sub-system level. Whenever an update of the schedule is required, sub-system managers (and the managers of large units inside sub-systems) update their project file and send them to the project manager for consolidation. With a global schedule made of 200 tasks the work involved in stitching the schedules together is important and errors prone. We really feel the need for a well engineered web-enabled management tool where managers at all levels can feed their inputs and that gives a clear view of the schedule evolution with time. Meanwhile, we are introducing a manual milestone tracking strategy to facilitate the control of the project.

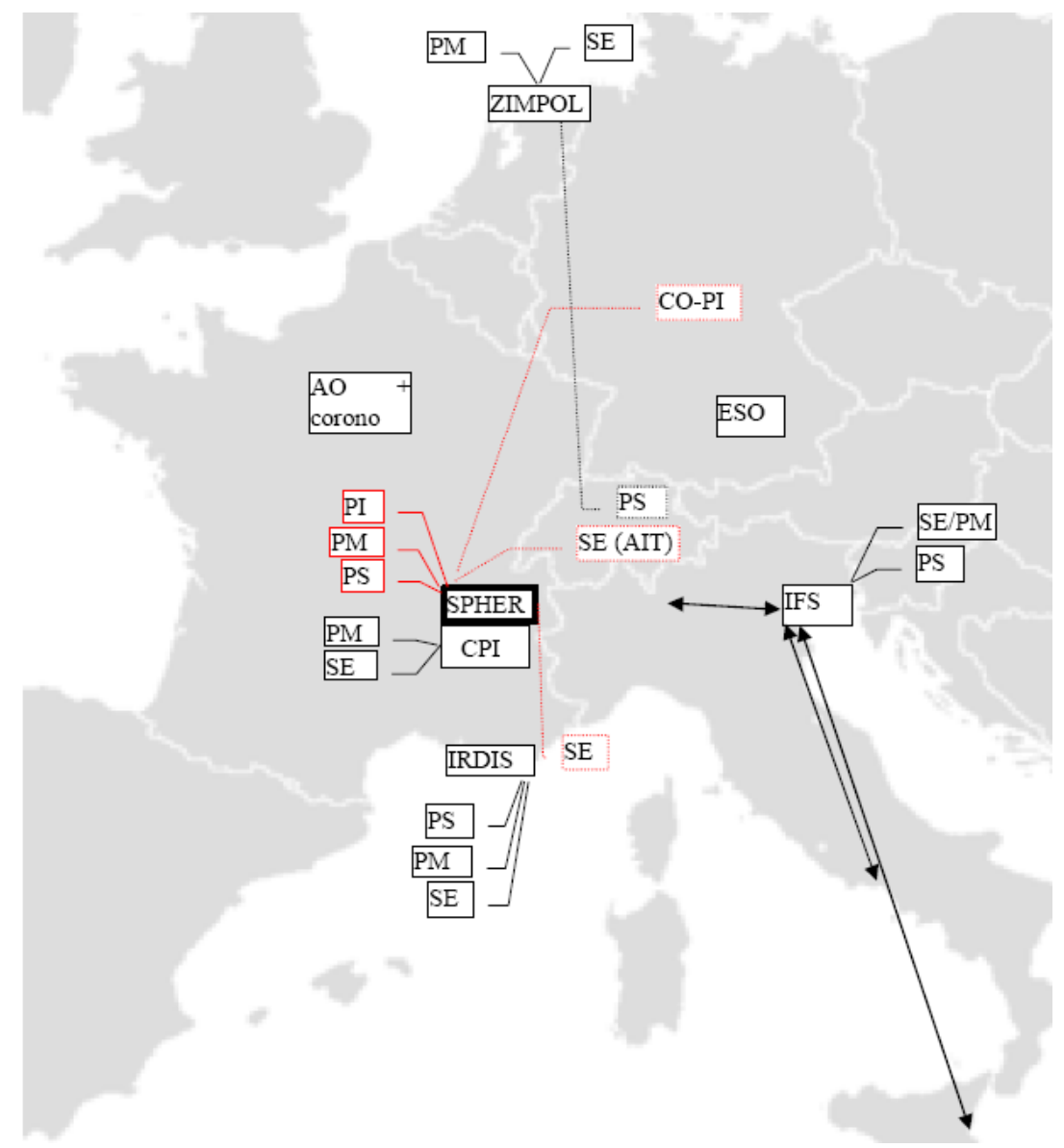

Figure 5. The SPHERE consortium in Europe 


\subsection{New technology developments}

One of the aspects that makes keeping the SPHERE schedule in check difficult is the fact that SPHERE will make use of a number of key high technology items that are only in their development phase. A new wavefront sensor integrated camera, a new detector read-out electronics, a new AO real time controller, and innovative toric mirrors. Inevitably, new technologies bring with them a certain level of risks, some of them being schedule risks. Moreover, apart from the torics, these developments are made or managed directly by ESO, and are not SPHERE-specific. Because of this situation SPHERE has no leverage on the way these developments are conducted from the technical, human resources and scheduling point of view.

It is worth noting that a high density high order deformable mirror and a specific electron multiplying CCD have been contracted to industrial partners and these developments have been a success.

\section{THE HUMAN RESOURCES FACTOR}

\subsection{Information flow}

With a project as involved as SPHERE with many interacting components and elaborate data reduction, maintaining an efficient information flow is very important. Different levels of formalism are used from the informal phone call to the formal change request that we could have to file to ESO. Email lists are also available to broadcast information to an interested group of persons. It is our experience that mail list are very efficient to broadcast information one-way. They are, however dangerous when the nature of the message is more speculative or when questions are raised, because several persons might provide an opinion or advice regarding the point raised, and this might spark a "discussion" via the mail list. The synthesis of the information spread over several email from different origins and interleaved with the original message turns out to be time consuming. A teleconference associated with factual minutes will be a more efficient use of participant's time. Success with videoconferencing has been limited at best because it appears that the images bring little advantage especially the little ones that are available, when a multi-point conference is conducted. In addition, setting up the conference is taking significant time until everybody is on line. The time is used more efficiently sharing the documents per email and reviewing them over the telephone

On a broader perspective; while it is important to maintain a sufficient level of information among the project members, it is neither desirable nor necessary that everybody knows everything in real time. We have been holding bimonthly system teleconferences and management teleconferences from very early in the project. The management teleconferences are efficient, often limited to the top-down flow of information from the PI and PM to the group and quickly solved organizational matters. The system teleconferences on the other hand are much lengthier and address a high level of detail in each of the sub-systems. They are obviously sub-optimal, because it is quite customary to ask someone a question and have him/her ask to repeat it because they had started going something else during the time other members were addressing details of another subsystem (attention deficit).

Defining the frequency and attendance of physical meetings has also proven to include lots of guesswork and to differ substantially from what was originally considered. There has been no system team meeting (the small committee) but there have been "system group" meetings, with all sub-system PS, SE and specialists of assemblies. These meetings are efficient at team building but they do mobilize everyone for two days for an objective contribution in the order of one hour.

At present, the head of the project (PI, PM, PS, SE and AIT resp) is initiating a series of "visits" of the four subsystems which are in fact mini reviews where all aspects of the projects are being scrutinized (design status, interfaces, manufacturing organisation, AIT, FDR documents, etc.). We are excited indeed to experiment what can be a different way of interacting with the sub-system teams, using everyone's time more efficiently.

\subsection{Responsibility and authority}

It is a fact that in university environment the organisation is very horizontal. It provides for quick circulation of ideas and a collaborative atmosphere. The fact that the consortium is very large provides for an even flatter organisation.

On the other hand, the project itself is organised in hierarchical layers.

The morphing of a flat organization into a vertical one is somewhat unnatural. It relies a lot on partners' goodwill and works well as long as no hard problems arise. If one does arise, in particular when there is a conflict in resources (personnel, funds, facilities), the project office is left with minimal authority to take executive decisions.

It is a well known fact that the responsibility given to someone to achieve success, must be coupled with the authority that will make that success possible. 


\subsection{On the "multiplex advantage"}

While multiplexing is certainly a big technological advantage, especially in modern astronomical instruments where using the full field information is now possible where a single point was the rule before, this advantage does not apply in the development of a project. We observe that the project is suffering from the fact that most team members are socalled "multiplexing" projects. While in the first case multiplexing refers to data collection happening concurrently in multiple channels; in the second case it refers to personnel switching tasks continually and doing them is pseudoparallel i.e. in series. This is detrimental to the project in the first place and to the global efficiency of the personnel in the institutes given the extra work and effort required to switch from one task to the next too often.

\section{DO WE NEED A NEW METHODOLOGY?}

\subsection{Limitations of the present system organisation and possible solutions}

- Establishment of requirements: The present policy is to accept requirements from the science side almost without limitation. This has important consequences on system complexity that can put the project in jeopardy in many ways: Consistency, SW development, integration, operational reliability. A critical review of the NAOS project would show that a lot of the system complexity added to the system has actually brought very little or no science return. A more space-like approach when complexity per se is considered a risk a clear risk management policy is established is probably called for. An efficient computer supported requirement flowdown (and back-up) system should be implemented as a matter of course in this kind of project

- Tracking of the requirements: Efficient methodology and/or tools are needed to constantly re-assess the consistency of the requirements and to balance the difficulty of achieving them between different parts of the system. To improve this situation without a change in paradigm, the optimal solution would probably be to have a system team fully dedicated to the project that can constantly re-visit the trades.

- $\quad$ Tracking the progress of the work: In a project of SPHERE's complexity, it has to be accepted that very few people have a complete view of the global issues. When reviewing the progress of the work internally, it is not necessary to involve all parties at the same time. Time would be better spent having the system team interact with each sub-system individually. Reviews with ESO every 3 month are good opportunities to update everybody on the progress of all parts of the system. We are initiating such a way of working with the system team performing detailed visits of the four sub-systems over the summer of 2008. If this experience is positive it will change the way system progress is monitored in the project.

- The good use of the talents: At all levels, we would benefit a lot from having personnel dedicated to the project. This would not only seep-up the work of individuals but this would considerably seep-up interactions between individuals where individual latencies add up. We even argue that from a broader perspective this would benefit individuals who would experience a lower level of stress. This is of course competing with the fact that in a university environment the pressure to publish will push towards a minor participation in several projects rather than a major one in a single project.

- Paying the right price: The way ESO is funding and rewarding consortia partners does not leave much freedom to rework the make-or-buy trade-offs. With ESO paying only part of the hardware, donating some parts and institutes contributing in paying the hardware and contributing 168 man-years of manpower (plus Ph.D. students and post-docs), the funding of the project is a very complicated balance. The fact that in European institutes do not have freedom to allocate there funds either to hiring or to contracting forces tem to use their own resources to do everyday engineering asks that would be better sub-contracted to industry. We argue that a funding model where the customer bears the "real total cost" of the project -or at least the bulk of it) would give a lot more flexibility in the way the make-or-buy trade can be made in the consortia and that would lead to faster development cycles.

All these areas and other that we have not listed here, can be considered as risk areas. These risk areas are often overlooked in a field like instrumentation where risk is essentially perceived as a possibility that the instrument does not reach its technological performance (scope) and leaves aside the other three constraints of a canonical project that are quality, time and budget.

\subsection{Where to go from there}

With the project running officially for over two years after the kick-off following the merger of the two original consortia, and with an instrument that is scheduled for first light in 3 years in Paranal, it does not appear feasible to implement large scale changes in the project structure or in the way the project is managed. However, changes in the methodology, to the extent that the project structure can accommodate then are certainly desirable. 
We can certainly implement schedule updates on a monthly basis at least at the level of the milestones.

We argue that the complexity of new VLT instrumentation does require at least a critical look in the way the management is put in place and that E-ELT instruments cannot avoid being done in a new management paradigm without running the major organisational risks.

From the days where instruments were built by astronomers themselves to today's academic multi-national consortia, astronomy has survived large changes of paradigms. Moving towards a more managed development of instruments will be mandatory move for next generation and will appear in retrospect as very wise; just as the VLT standards are today. As matter of fact, for the space-borne observatories, astronomy has already been working in a more formal environment for decades.

\section{CONCLUSION}

We have presented here the status of SPHERE with an emphasis on the project organization. This very exciting and very promising project is complex not only from the technical point of view but also from the point to view of the project management. With 11 institutes and even more sites, with several high technology devices under development, with personnel that is working in time-share mode, keeping the project on track requires a constant effort and a high flexibility from the project head.

This project organization model with several academic institutes partnering and each funding its share of the project is not a good match for development of today's large instruments. Large organizations like ESO should put a working group in place to draw the best possible guidelines for the organization of today's and tomorrow's complex ground based instruments projects. Opportunities should be given to potential actors in these projects to be trained and acquire the proper mindset.

\section{REFERENCES}

1. Jean-Luc Beuzit; Markus Feldt, Kjetil Dohlen; David Mouillet, Pascal Puget, Francois Wildi., "SPHERE: a 'Planet Finder' instrument for the VLT", in Ground-based and Airborne Instrumentation for Astronomy II, SPIE 7014-42 (2008)

2. Mouillet, D.; Lagrange, A. M.; Beuzit, J.-L.; Moutou, C.; Saisse, M.; Ferrari, M.; Fusco, T.; Boccaletti, A., "High Contrast Imaging from the Ground: VLT/Planet Finder," in Extrasolar Planets: Today and Tomorrow, JeanPhilippe Beaulieu, Alain Lecavelier des Etangs and Caroline Terquem (eds), ASP Conf. Proc. 321, 2004, p.39.

3. M. Feldt, T. Henning, S. Hippler, M. Turatto, R. Neuhaeuser, H. M. Schmid, R. Waters "The CHEOPS project: CHaracterizing Exo-planets by Opto-infrared Polarimetry and Spectroscopy" in Exploring the cosmic frontiers: Astrophysical instruments for the 21st century, Proc. ESO Astrophysics Symposium (2005).

4. Cyril Petit, Jean-Marc Conan, Thierry Fusco; Enrico Fedrigo; Caroline Kulcsar, Henri-Francois Raynaud, "Optimization of the control laws for the SPHERE XAO system, in Adaptive optics systems, SPIE 7015-48 (2008)

5. Anthony Boccaletti "Prototyping achromatic coronagraphs for exoplanet characterization with SPHERE", in Adaptive Optics Systems, SPIE 7015-46 (2008)

6. S. Desidera, R. Gratton, R. Claudi, J. Antichi, D. Mesa, M. Turatto,; P. Bruno, E. Cascone,; V. De Caprio,; E. Giro; S. Scuderi; M. Feldt, A. Pavlov, K. Dohlen, J-L Beuzit, D. Mouillet, P. Puget, F. Wildi, "Calibration and data reduction for planet detection with SPHERE-IFS", in Ground-based and Airborne Instrumentation for Astronomy II, SPIE 7014-127 (2008)

7. Riccardo U. Claudi, Massimo Turatto, Raffaele G. Gratton, Jacopo Antichi,; Enrico Cascone,; Vincenzo De Caprio,; Silvano Desidera, Dino Mesa, Salvatore Scuderi, "SPHERE IFS: the spectro differential imager of the VLT for exoplanets search", in Ground-based and Airborne Instrumentation for Astronomy II, SPIE 7014-119 (2008)

8. Christian Thalmann, Hans M. Schmid, Anthony Boccaletti, David Mouillet, Kjetil Dohlen, Ronald Roelfsema, Marcel Carbillet, Daniel Gisler, Jean-Luc Beuzit, Markus Feldt, Raffaele Gratton, Franco Joos, Christoph U. Keller, Jan Kragt, Johan H. Pragt, Pascal Puget, Florence Rigal, Frans Snik, Rens Waters, Francois Wildi, "SPHERE ZIMPOL: Overview and performance simulation", in Ground-based and Airborne Instrumentation for Astronomy II, SPIE 7014-120 (2008)

9. K. Dohlen; J.-L. Beuzit; M. Feldt; D. Mouillet; P. Puget; J. Antichi; A. Baruffolo; P. Baudoz; A. Berton; A. Boccaletti; M. Carbillet; J. Charton; R. Claudi; M. Downing; Ch. Fabron; Ph. Feautrier; E. Fedrigo; T. Fusco; J.-L. Gach; R. Gratton; N. Hubin; M. Kasper; M. Langlois; A. Longmore; C. Moutou; C. Petit; J. Pragt; P. Rabou; G. Rousset; M. Saisse; H.-M. Schmid; E. Stadler; D. Stamm; M. Turatto; R. Waters; F. Wildi, "SPHERE: A planet 
finder instrument for the VLT" in: Ground-based and Airborne Instrumentation for Astronomy, Ian S. McLean, Masanori Iye, eds, Proc SPIE 6269 (2006)

10. . Bacon, R, "The integral field spectrograph TIGER: results and prospects. In: 3D Optical Spectroscopy Methods in Astronomy, G. Comte, M. Marcelin (eds), ASP Conf. Series 71, 1995, p. 239

11. Carbillet, M.; Verinaud, Ch.; Guarracino, M.; Fini, L.; Lardiere, O.; Le Roux, B.; Puglisi, A. T.; Femenia, B.; Riccardi, A.; Anconelli, B.; Correia, S.; Bertero, M.; Boccacci, P., "CAOS: a numerical simulation tool for astronomical adaptive optics (and beyond)," In: Advancements in Adaptive Optics. Proc. SPIE 5490, pp. 637-648 (2004).

12. A. Blanc, T. Fusco, M. Hartung, L. M. Mugnier et G. Rousset, "Calibration of NAOS and CONICA static aberrations. Application of the phase diversity technique," Astron. Astrophys., 399 (2003), pp. 373-383.

13. Thatte, N., Abuter, R., Tecza, M., Nielsen, E.L., Clarke, F.J., Close, L.M., 2007, MNRAS, 378, 1229, "Very high contrast integral field spectroscopy of $\mathrm{AB}$ Doradus C: 9-mag contrast at 0.2arcsec without a coronagraph using spectral deconvolution" 